\title{
USO DE CLAR EN FASE INVERSA PARA LA DETERMINACIÓN DEL CONTENIDO DE FORMALDEHÍDO LIBRE EN ESMALTES DE UÑNA
}

\author{
María Maldonado-Santoyo ${ }^{1}$, Erika Hernández-Guerrero², Gladys Morales- \\ López ${ }^{3}$, Javier Antonio Arcibar-Orozco ${ }^{4}$, Juliette-Alexandra Lambert ${ }^{4}$, \\ Jennifer Alexis Bañuelos-Díaz ${ }^{4}$ \\ ${ }^{1}$ Profesor-Investigador en el área de Química Ambiental, Dirección de Investigación, Posgrado y Capacitación, \\ Centro de Innovación Aplicada en Tecnologías Competitivas (CIATEC), León, Guanajuato, México. Correo \\ electrónico:msantoyo@ciatec.mx \\ ${ }^{2}$ Licenciatura en Ingeniería Química Sustentable, División de Ciencias e Ingenierías, Universidad de Guanajuato, \\ México \\ ${ }^{3}$ Profesor Investigador en el área de Ingeniería en Agrotecnología, Universidad Politécnica del Bicentenario, \\ Guanajuato, México \\ ${ }^{4}$ Investigador del Centro de Innovación Aplicada en Tecnologías Competitivas (CIATEC), León, Guanajuato, México
}

Fecha de recibido: 20 de septiembre del 2016 Fecha de aprobado: 22 de febrero del 2017

Cómo citar este artículo: M. Maldonado-Santoyo, E. Hernández-Guerrero, G. Morales-López, J. A. Arcibar-Orozco, J. A. Lambert, J. A. Bañuelos-Díaz, "Uso de CLAR en fase inversa para la determinación del contenido de formaldehído en esmaltes de uñas", Ingeniería Solidaria, voL. 13, n. ${ }^{\circ}$ 22, mayo de 2017, pp. 27-37. doi: http://dx.doi.org/10.16925/in.v13i22.1750

Resumen. Introducción: este artículo es producto de la investigación titulada "Uso de CLAR en fase inversa para la determinación del contenido de formaldehído libre en esmaltes de uñas", desarrollada en el 2015 en el Centro de Innovación Aplicada en Tecnologías Competitivas (CIATEC). Metodología: se determinó el contenido de formaldehído libre en 50 muestras de esmaltes para uñas de diversas marcas comercializadas en León, Guanajuato, México. El tratamiento de muestras se realizó de acuerdo con el método Iso 17226-1:2003, con ligeras modificaciones: se pesaron $2 \pm 0,1 \mathrm{~g}$ de esmaltes en matraces Erlenmeyer de $100 \mathrm{ml}$, se adicionaron $40 \mathrm{ml}$ de dodecil sulfonato de sodio al 0,1\%. Las muestras fueron entonces tapadas y se dejaron en baño María a $40{ }^{\circ} \mathrm{C}$ con agitación constante durante 60 minutos; posteriormente se filtraron y se tomaron alícuotas de cada muestra para su derivatización con 2,4-dinitrofenilhidracina al 0,3\% durante 60 minutos. La determinación analítica se realizó por CLAR en fase inversa con detección por arreglo de diodos. Resultados: se obtuvo como resultado que todas las muestras de esmaltes para uñas analizadas estuvieron por debajo del límite normativo de contenido de formaldehído libre en cosméticos de $0,2 \%$. Conclusiones: se obtuvo información de relevancia toxicológica sobre el formaldehído libre en los esmaltes de uñas, además de comprobar la versatilidad y robustez del método de prueba.

Palabras clave: CLAR, DNFH, esmalte de uñas, formaldehído. 


\title{
Use of ReVersed-Phase hPlC FOR The Determination of Free Formaldehyde Content in NaIl Polishes
}

\begin{abstract}
Introduction: This article derives from the research titled Use of reversed-phase HPLC for the determination of free formaldehyde content in nail polishes, conducted in 2015 at the Center for Applied Innovation in Competitive Technologies (CIATEC). Method: The free formaldehyde content was determined in 50 samples of nail polishes of various brands marketed in León, Guanajuato, Mexico. Samples were treated according to the Iso 17226-1:2003 method, with slight modifications: $2 \pm 0.1 \mathrm{~g}$ of nail polishes were weighed in $100 \mathrm{ml}$ Erlenmeyer flasks, $40 \mathrm{ml}$ of $0.1 \%$ sodium dodecyl sulfonate were added; the samples were then capped and left in a water bath at $40{ }^{\circ} \mathrm{C}$ under constant stirring for 60 minutes; they were subsequently filtered, and aliquots of each sample were taken for derivatization with $0.3 \%$ 2,4-dinitrophenylhydrazine for 60 minutes. The analytical determination was achieved by reversed-phase HPLC-diode array detection. Results: All nail polish samples tested were below the regulatory limit of $0.2 \%$ free formaldehyde content in cosmetics. Conclusions: We obtained toxicologically relevant information on free formaldehyde in nail polishes, in addition to checking the versatility and robustness of the test method.
\end{abstract}

Keywords: HPLC, DNFH, nail polish, formaldehyde.

\section{USO DE CLAR EM FASE INVERSA PARA A DETERMINAÇÃO DO CONTEÚDO DE FORMALDEÍDO LIVRE EM ESMALTES DE UNHAS}

Resumo. Introdução: este artigo é produto da pesquisa intitulada "Uso de CLAR em fase inversa para a determinação do conteúdo de formaldeído livre em esmaltes de unhas”, desenvolvida em 2015 no Centro de Inovação Aplicada em Tecnologias Competitivas (Ciatec). Metodologia: determinou-se o conteúdo de formaldeído livre em 50 amostras de esmaltes para unhas de diversas marcas comercializadas em León, Guanajuato, México. O tratamento de amostras foi realizado de acordo com o método Iso 17226-1:2003, com leves modificações: foram pesados 2 $\pm 0,1 \mathrm{~g}$ de esmaltes em frascos Erlenmeyer de $100 \mathrm{~mL}$, adicionados $40 \mathrm{~mL}$ de dodecil sulfonato de sódio a $0,1 \%$; as amostras foram então tampadas e deixadas em banho-maria a $40{ }^{\circ} \mathrm{C}$ com agitação constante durante 60 minutos; em seguida, foram filtradas e tomadas alíquotas de cada amostra para sua derivatização com 2,4- dinitrofenilhidrazina a 0,3 \% durante 60 minutos. A determinação analítica foi realizada por CLAR em fase inversa com detecção por ajuste de diodos. Resultados: obteve-se como resultado que todas as amostras de esmaltes para unhas analisadas estiveram abaixo do limite normativo de conteúdo de formaldeído livre em cosméticos de 0,2 \%. Conclusões: obteve-se informação de relevância toxicológica sobre o formaldeído livre nos esmaltes de unhas, além de comprovar a versatilidade e a solidez do método de teste.

Palavras-chave: CLAR, DNFH, esmalte de unhas, formaldeído. 


\section{Introducción}

El formaldehído es el aldehído que se encuentra con más frecuencia en el ambiente [1]. Es un gas inflamable e incoloro que tiene un olor penetrante característico (EPA, 2016), es muy reactivo y polimeriza fácilmente a temperatura ambiente [2]. Se puede adquirir comercialmente como una solución acuosa del 30-50\% en peso [2]; algunos de los nombres derivados del formaldehído que comúnmente aparecen en las etiquetas comerciales de los productos son ácido fórmico, formalina, óxido de etileno, metanaldehído, oxometano o formol [3]. Pequeñas cantidades de formaldehído son producidas de forma natural por los humanos, las plantas y los animales. Es uno de los compuestos orgánicos básicos utilizados en la industria química por su poder antiséptico, desinfectante y conservante. Es muy empleado en una gran cantidad de productos tales como maderas, resinas, comida [4], fertilizantes, papel, resinas de ureaformaldehído, antisépticos, textiles [5], medicamentos y diversos productos de limpieza [2], así como de uso cosmético [2], por ejemplo, cremas, champús, locio-nes, gel de baño, perfumes, aerosoles, pastas de dientes, alisadores para el cabello y esmaltes de uñas, entre otros. Son estos últimos los que se destacan por los altos niveles de formaldehído, el cual es adicionado para dar un aspecto brillante, plastificado, aumentar la capacidad de secado y otorgar sus propiedades de conservante [3, 6-9], incrementando así el tiempo de vida del producto.

El vapor y las soluciones de formaldehído libre son corrosivas e irritantes cutáneos, nasales y oculares para los seres humanos cuando están expuestos a concentraciones superiores al $2 \%$; derivado de esto, en el 2004 el formaldehído fue declarado como carcinogénico para el hombre en la categoría Grupo 1 por la Organización Mundial de la Salud (OMs).

De acuerdo con la Food and Drugs Administration (FDA) [3], el contenido de formaldehído libre utilizado como conservador permitido en productos cosméticos debe ser menor al 0,2 \% (2 mg/g) [3], mientras que, en productos cosméticos destinados al endurecimiento de uñas, seautoriza en concentraciones de hasta un $5 \%$ (50 mg/g) [3] [10]. En concentraciones menores al $1 \%(10 \mathrm{mg} / \mathrm{g})$ se emplea en bienes de consumo tales como productos caseros de limpieza, líquidos lavaplatos, suavizante de telas, agentes para el cuidado del calzado, champús, ceras para automóvil y agentes para la limpieza de alfombras [2].
Una gran variedad de técnicas analíticas instrumentales han sido empleadas para la determinación de formaldehído en diferentes tipos de muestras. La mayoría de ellas se basan en la espectrofotometría [1, 4]. Otros métodos incluyen la colorimetría, fluorimetría, cromatografía líquida de alta resolución (CLAR) $[1,4,11,12]$ cromatografía gaseosa [4], polarografía, detección infrarroja y tubos detectores de gases [2] —estos dos últimos utilizados en el monitoreo de las atmósferas en los lugares de trabajo-, espectrometría de masas con dilución isotópica [6], fibra óptica, cromatografía líquida acoplada a espectrometría de masas [11], y electroforesis capilar [4]. Es de importancia considerar que, dependiendo del método de análisis se pueden lograr sensibilidades de hasta $0,12 \mathrm{ng} /$ mL [4] o menores, y cómo de forma complementaria dentro de este contexto, el método de muestreo y la preparación de muestra antes del análisis por alguna de las técnicas analíticas mencionadas, es un paso importante en el proceso analítico para la determinación de cantidades traza de todo analito que se desea analizar, así como también para la calidad de los resultados obtenidos [2, 4].

De todas las técnicas mencionadas, la cromatografía de líquidos de alta resolución es, por su alta sensibilidad y selectividad, rápida y fácil de usar [4, 11] en determinaciones analíticas, así como la técnica más utilizada para separar el formaldehído de posibles interferencias y mejorar el límite de detección. Por otro lado, con respecto a lo que a tratamiento de muestra se refiere, es el procedimiento de derivatización que se basa en la reacción de las muestras con la 2,4-dinitrofenilhidracina (DNFH) por su selectividad de reacción con aldehídos y cetonas, al formar compuestos coloreados estables de tipo hidrazonas [4, 11-15], el utilizado con más frecuencia.

El presente estudio reporta la determinación del contenido de formaldehído libre en cincuenta muestras representativas de esmaltes de uñas comercializadas en León, Guanajuato, México. Se basa en el método Iso 17226-1:2003, con ligeras modificaciones, separación por CLAR en fase inversa y detección por arreglo de diodos, lo que permitió obtener información analítica. Por otro lado, es importante comentar que en la revisión de la literatura se encontró poca información directamente relacionada para este tipo de muestras. La mayoría de los estudios encontrados centran su atención en muestras tales como perfumes, 
champús, cremas, alimentos, bebidas alcohólicas, fármacos, y en el aire, entre otros; o bien, en los efectos toxicológicos de exposición al formaldehído en el ambiente, razón por la cual este trabajo aporta información, tanto de relevancia toxicológica, como analítica específica para este tipo de muestras, lo que a futuro servirá para un estudio de mayor profundidad y/o alcance.

También es importante comentar que el enfoque del trabajo no es la evaluación del contenido de formaldehído por marca, sino contar con una muestra representativa que provea de información sobre el uso del formaldehído en los esmaltes de uñas comercializados en la ciudad, indistintamente de la marca comercial.

\section{Materiales y métodos}

\subsection{Muestras}

Un total de 50 muestras de esmaltes para uñas de igual o diferente marca comercializadas en León se obtuvieron en tiendas de autoservicio, tiendas departamentales y de belleza, supermercados o por catálogo. Algunas de las marcas analizadas fueron Color naturals, Im, Del baúl de la abuela, Profussión, Amour, Principessa, Renova, Equate, Uña maravilla, Avon, Holliwood cosmetics, Fulgurance, Apple cosmetic, Alarga, Mabelle, Queen, Saniye, Olé!, Angelissima, Bissú, Delish, Mia Secret, Yuri, Sweet color, Gelden, China glaze, Mabelita, Jafra, Color fun, Elf, Seche, Crystal nails, OPI, Revlon, Essie, Nabi, Gem Crush, Max Factor y Amorette.

\subsection{Reactivos y disoluciones}

Todos los reactivos utilizados fueron grado analítico. Agua desionizada (Karal/cas:7732-18-5/<1,5 $\mu$ mhos) fue utilizada en la preparación de los estándares y muestras. El acetonitrilo (ACN) fue grado HPLC (Karal/99,8\%/cas:75-05-8); la solución de dodecil sulfato de sodio (us Biological/99\%/cas:151-21-3) se preparó al 0,1\% en agua; la 2,4-dinitrofenilhidracina (DNFH) (Fluka/97\%/cas:119-26-6) se preparó al $0,3 \%$ en ácido fosfórico concentrado (Quimir/85\%/ cas:007664-38-2), y el estándar certificado de formaldehído fue de Accustandard a una concentración 1000 $\mu \mathrm{g} / \mathrm{mL}$ en agua (M-8315-02).

\subsection{Recristalización de la dinitrofenilhidracina}

La recristalización de la 2,4-dinitrofenilhidracina se realizó de acuerdo con el método EPA 8315A [17], con ligeras modificaciones: se preparó una solución saturada de DNFH en $200 \mathrm{~mL}$ de acetonitrilo y se calentó a ebullición durante una hora. Transcurrido este tiempo, la solución se dejó enfriar gradualmente hasta una temperatura de $60^{\circ} \mathrm{C}$, manteniendo esta temperatura hasta la evaporación del solvente (aprox. $95 \%$ ). Posteriormente, se decantó la solución y los cristales de DNFH fueron lavados tres veces con acetonitrilo y secados en atmósfera de argón. Este proceso se repitió dos veces más hasta eliminar toda impureza de formaldehído en la DNFH, y se almacenaron en desecador en frasco ámbar.

\subsection{Instrumentación}

El análisis cromatográfico se realizó en un cromatografo de líquidos de alta resolución de Agilent Technologies, modelo 1100 con bomba cuaternaria y detector con arreglo de diodos. Para la separación analítica se empleó una columna de fase inversa Zorbax Eclipse xDB8-c8 (150x4,6 mm,0,5 $\mu \mathrm{m})$; la fase móvil fue acetonitrilo: agua $(53: 47 \mathrm{v} / \mathrm{v})$ en modo isocrático con un flujo de $1,00 \mathrm{~mL} / \mathrm{min}$. El volumen de muestra inyectada fue de $20 \mu \mathrm{L}$ con una temperatura de $23^{\circ} \mathrm{C}$ en la entrada en la columna. La detección del formaldehído se realizó a $360 \mathrm{~nm}[4,18-20]$. El análisis de datos se llevó a cabo en el programa Chemstation.

\subsection{Extracción de formaldehído}

La extracción del contenido de formaldehído libre en las muestras de esmaltes para uñas se realizó de acuerdo con el método iso 17226-1:2003 [16], con ligeras modificaciones: se pesaron $2 \pm 0,1 \mathrm{~g}$ de muestra de esmalte de uñas (sin tratamiento previo), en matraces Erlenmeyer de $100 \mathrm{~mL}$, se adicionaron $40 \mathrm{~mL}$ de dodecil sulfonato de sodio al 0,1\% (precalentado a $40^{\circ} \mathrm{C}$ ); así, entonces, las muestras fueron cerradas y colocadas en baño María a $40^{\circ} \mathrm{C}$ con agitación constante durante $60 \pm 2$ minutos. Transcurrido este tiempo, las muestras se filtraron en papel whatman $n .^{\circ} 41$, y se dejaron enfriar a temperatura ambiente. De forma general, para el proceso de derivatización se tomaron alícuotas de 
$800 \mu \mathrm{L}$ de cada muestra filtrada, a la cual se le adicionaron $700 \mu \mathrm{L}$ de ACN y $100 \mu \mathrm{L}$ de DNFH al 0,3\%; se mezclaron y se dejaron derivatizando durante 60 minutos antes de su análisis cromatográfico; a la par, también se analizaron el blanco y muestras de esmaltes adicionadas con estándar de formaldehído a una concentración de $0,188 \mu \mathrm{g} / \mathrm{ml}$, con la finalidad de determinar la eficiencia de recuperación del proceso de extracción. Todas las muestras se analizaron el mismo día y por duplicado.

\section{Resultados}

\subsection{Selectividad}

La selectividad del método de análisis utilizado en este estudio se puede observar en la figura 3 , en la que se presentan los cromatogramas obtenidos para una muestra de esmalte con contenido bajo de formaldehído, otra muestra con contenido alto de formaldehído y un blanco de método, detectados a $360 \mathrm{~nm}$ y libres de interferencias provenientes de la matriz de estudio (esmaltes de uñas) [21].

\subsection{Intervalo de trabajo}

El intervalo lineal de trabajo fue obtenido mediante la calibración externa con nueve niveles de concentración de formaldehído $(0,005,0,010$, $0,05,0,10,0,40,0,80,1,20,1,60$ y $2,00 \mu \mathrm{g} / \mathrm{mL})$. Los resultados obtenidos en la calibración se presentan en la figura 1, la ecuación obtenida fue $\mathrm{y}=9067,6 \mathrm{x}$ $+10,697$ con un coeficiente de regresión de 0,9993, siendo " $\mathrm{x}$ ": la concentración de formaldehído, $y$ " $y$ ": la respuesta dada por el equipo de medición en miliunidades de absorbancia (mAU). El límite de detección alcanzado fue de $0,9 \mathrm{ng} / \mathrm{mL}$, el cual fue estimado por análisis del blanco calculado como la relación señal/ruido $(3: 1)[4,11,15,22]$; por su parte, el límite de cuantificación para este estudio se consideró el primer nivel de la curva de calibración, es decir, $0,005 \mu \mathrm{g} / \mathrm{mL}$ [21,23].

Para la identificación de los analitos de interés (DNFH y formaldehído) se realizó con base a su tiempo de retención, obtenido en el análisis cromatográfico al ser inyectados en forma individual. En la figura 2 se presentan los cromatogramas obtenidos por CLAR en fase inversa para el blanco y los estándares de formaldehido a las concentraciones de $0,1,1,2$

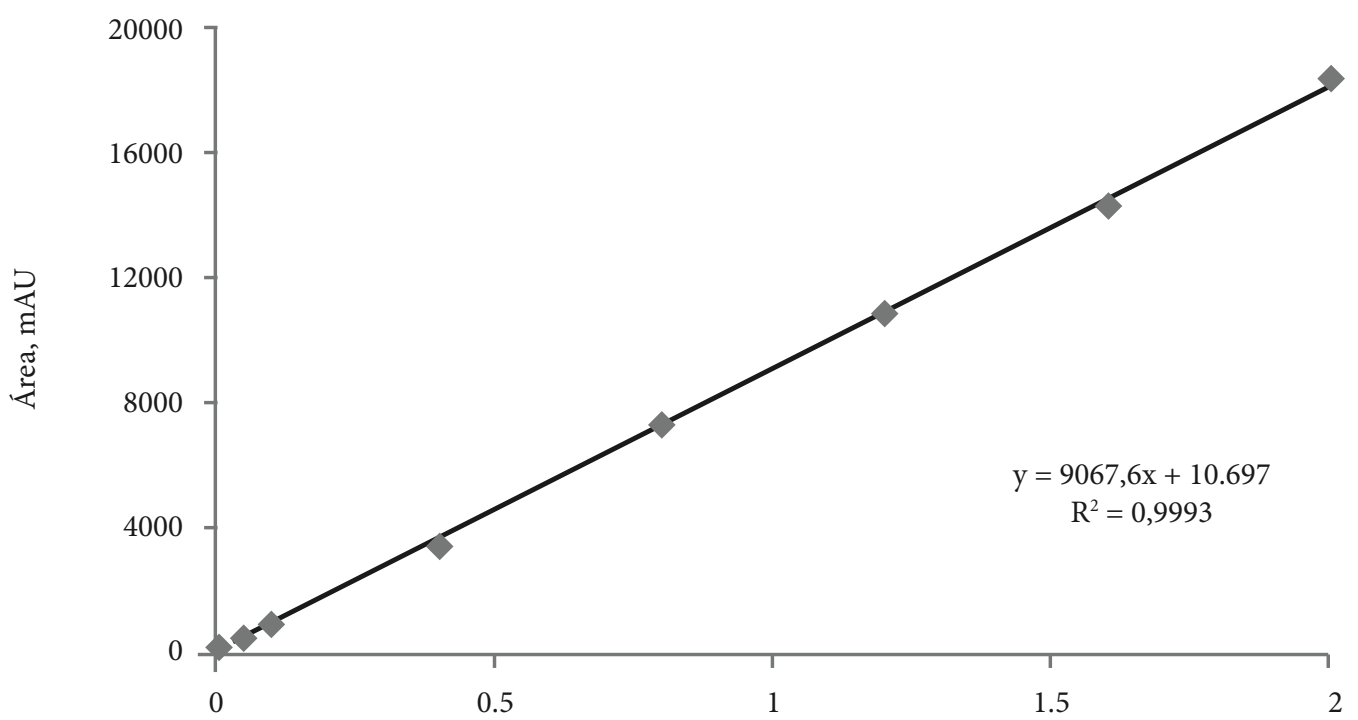

Formaldehído, $\mu \mathrm{g} / \mathrm{mL}$

Figura 1. Calibración externa obtenida por ClAR para nueve niveles de concentración del estándar de formaldehído $(0,005 ; 0,010$; $0,05 ; 0,10 ; 0,40 ; 0,80 ; 1,20 ; 1,60$ y $2,00 \mu \mathrm{g} / \mathrm{ml})$

Fuente: elaboración propia 


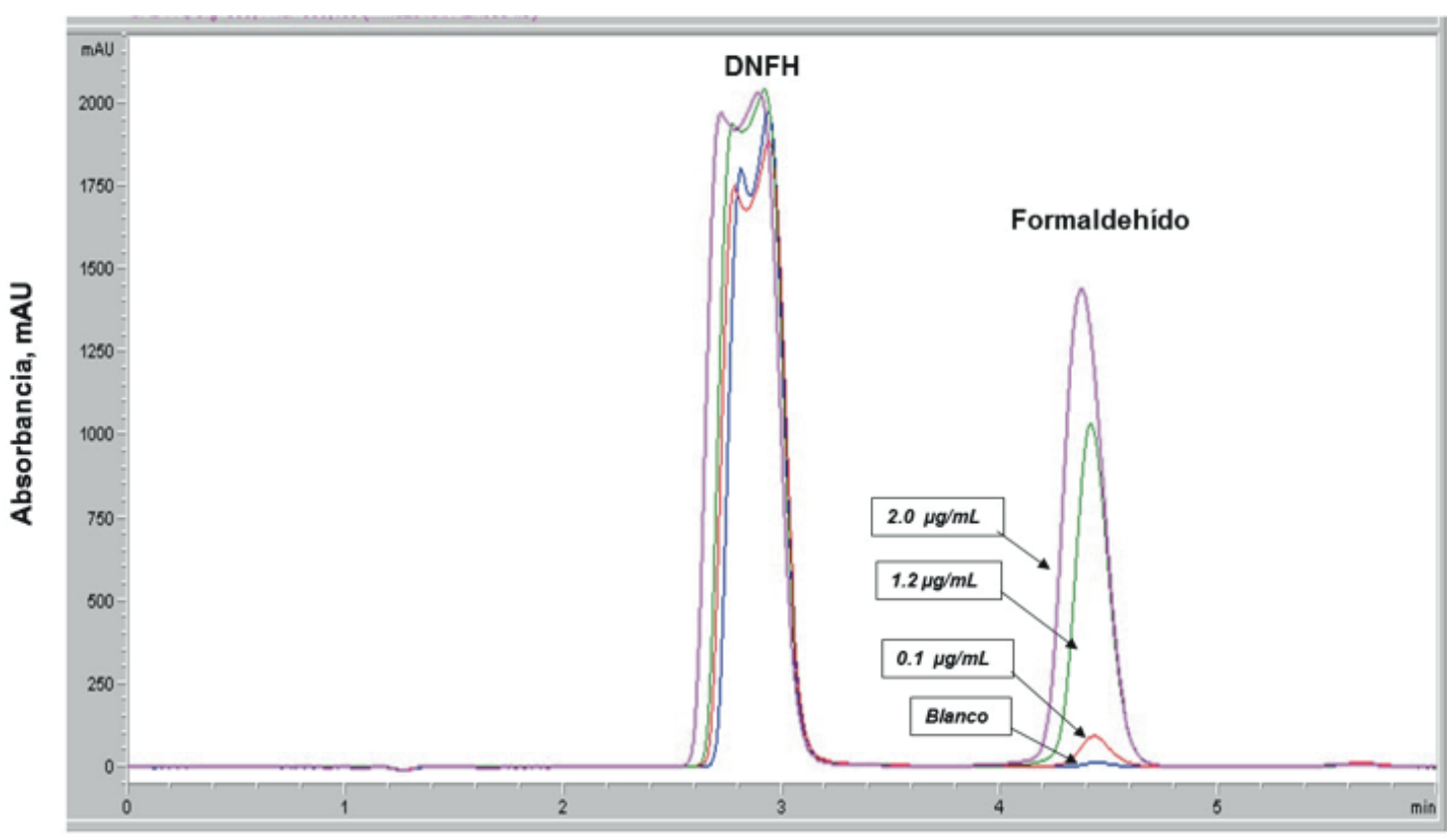

Tiempo, $\min$

Figura 2. Cromatogramas obtenidos por Clar para el blanco y diferentes concentraciones del estándar de formaldehído: 0,1 $\mu \mathrm{g} /$ $\mathrm{mL}, 1,2 \mu \mathrm{g} / \mathrm{mL}$ y $2,0 \mu \mathrm{g} / \mathrm{mL}$

Fuente: elaboración propia

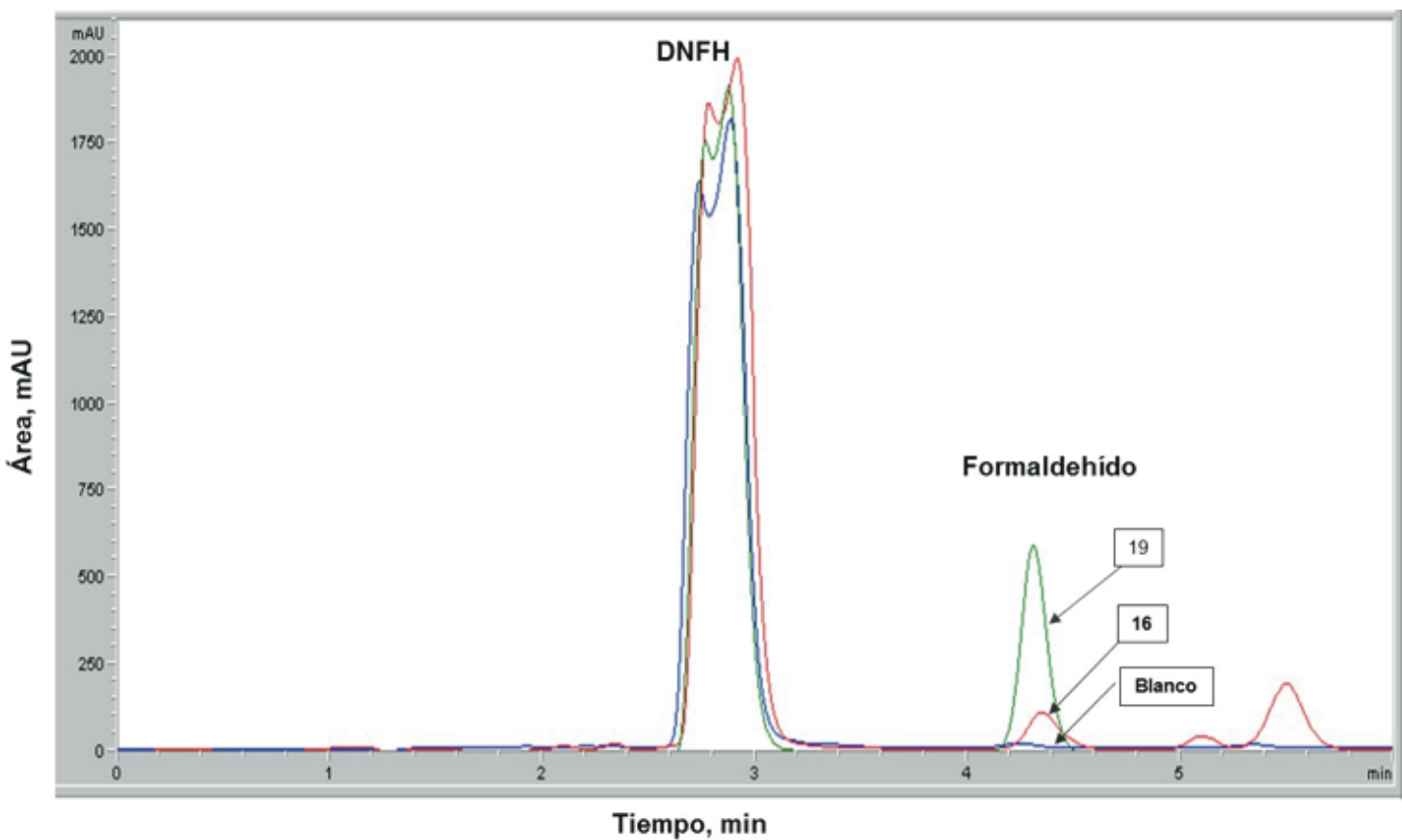

Figura 3. Ejemplo de cromatogramas obtenidos por CLAR en el análisis de formaldehído en blanco, muestra 16 y muestra 19 de los esmaltes de uñas analizadas

Fuente: elaboración propia 
y 2,0 $\mu \mathrm{g} / \mathrm{mL}$. El tiempo de elución fue 3,82 min para la DNFH, y de 4,32 min para el formaldehído.

Por otro lado, en la figura 3 se presentan los cromatogramas obtenidos por CLAR en el análisis del contenido de formaldehído libre en las muestras de esmaltes de uñas correspondientes al blanco, muestra 16 y muestra 19. En ellas se puede apreciar similar comportamiento y tiempo de elución para la DNFH y el formaldehído, con respecto a los cromatogramas obtenidos en el estándar de formaldehído. Picos pequeños pueden observarse en las soluciones del blanco, esto derivado de que el formaldehído está presente en forma gaseosa en el aire [10]. Al comparar los tiempos de respuesta, son similares a los reportados por Wang et al. [11] en el análisis de formaldehído mediante CLAR en muestras de cerveza; al de Xu et al. [4] en muestras de comida, y al de Herrington et al. [24] en aire utilizando sorbentes sólidos recubiertos con DNFH. Esto permite apreciar la estabilidad de los compuestos de tipo hidrazona que, indistintamente de la matriz de obtención del formaldehído, su elución en la columna se mantiene de forma muy similar.

\subsection{Precisión}

La precisión de las determinaciones del contenido de formaldehído libre en las muestras de esmaltes fue evaluado como el porcentaje de diferencia relativa (RPD) de la concentración medida en las muestras (no adicionadas) por duplicado, mediante la ecuación 1 [25]:

$$
R P D=\frac{|C 1-C 2|}{\frac{C 1+C 2}{2}} \times 100
$$

Donde:

$c_{1}$ : concentración medida de la primer alícuota de muestra

$\mathbf{c}_{2}$ : concentración medida de la segunda alícuota de muestra

RPD: porcentaje de diferencia relativa

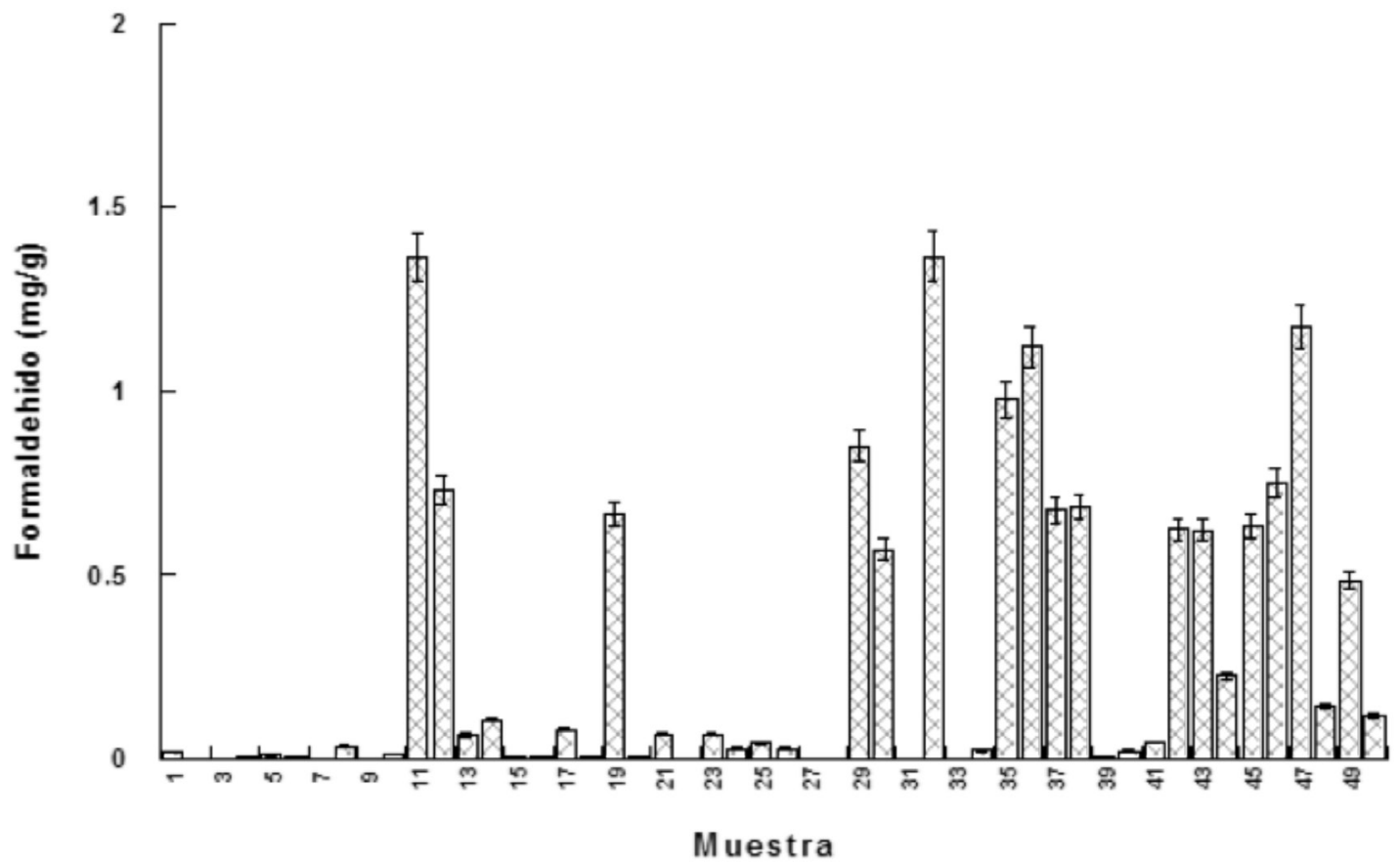

Figura 4. Contenido de formaldehído libre ( $\mathrm{mg} / \mathrm{g})$ determinado en cincuenta muestras comerciales de esmaltes de uñas Fuente: elaboración propia 


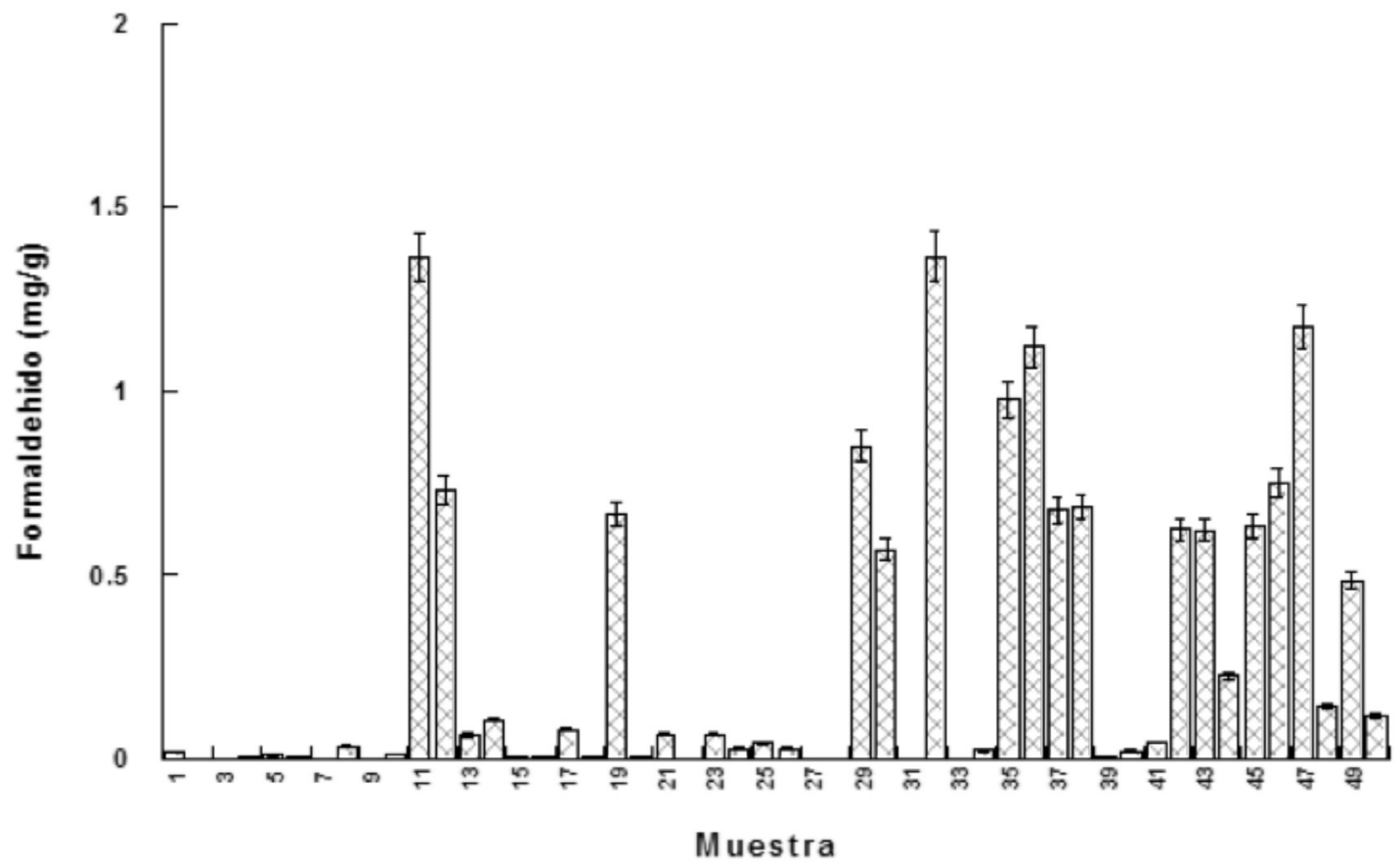

Figura 4. Contenido de formaldehído libre $(\mathrm{mg} / \mathrm{g})$ determinado en cincuenta muestras comerciales de esmaltes de uñas Fuente: elaboración propia

Los valores de RPD obtenidos para las 50 muestras analizadas fueron menores al $5 \%$. En la figura 4 se presentan gráficamente los resultados del contenido de formaldehído determinado en las 50 muestras de esmaltes analizadas con una desviación estándardel $5 \%$. Donde el contenido de formaldehído para las muestras $1-10,13,15-18,30$ y $39-41$ fue: $<0,1 \mathrm{mg} / \mathrm{g}$; de 0,1 a 0,5 $\mathrm{mg} / \mathrm{g}$ para las muestras $14,44,48-50$; en el intervalo de 0,5 a $1,0 \mathrm{mg} / \mathrm{g}$ se encontraron las muestras 12,19 , $29,30,35,37,38,42,43,45$ y 46 ; y las muestras 11 , 32,36 y 47 se encontraron en el intervalo de 1,0 a $1,5 \mathrm{mg} / \mathrm{g}$. De forma general, se puede apreciar que el contenido de formaldehído libre en todas los esmaltes de uñas analizados en este trabajo se encuentran por debajo de $1,5 \mathrm{mg} / \mathrm{g}$, cumpliendo de esta manera con el valor normativo permitido de $2 \mathrm{mg} / \mathrm{g}(0,2 \%)$ en cosméticos, con lo cual se observa quela aplicación del formaldehído para estas muestras de esmaltes es conservador.

\subsection{Recuperación}

Para la evaluación de la eficiencia de recuperación del formaldehído con el procedimiento aplicado en este trabajo [16], se efectuaron análisis a seis muestras diferentes de los esmaltes de uñas (M2, M7, M17, м22, м37 y м42), adicionando cada una de ellas una concentración conocida de formaldehído de $0,188 \mu \mathrm{g} / \mathrm{mL}$. Todos los análisis se realizaron por duplicado. El porcentaje de recuperación fue determinado conforme a la ecuación 2 [25].

$\% R=\frac{C s-C u}{C n} \times 100$

Donde:

$\mathrm{C}_{\mathrm{s}}$ : concentración medida de la alícuota de muestra adicionada

$\mathrm{C}_{\mathrm{u}}$ : concentración medida de la alícuota de muestra sin adicionar

$\mathrm{C}_{\mathrm{n}}$ : concentración nominal (teórica) de la muestra adicionada

\%R: porcentaje de recuperación

En la tabla 1 se presentan los valores de recuperación obtenidos para las siete muestras de esmaltes adicionadas con estándar de formaldehído, a una concentración de $0,188 \mu \mathrm{g} / \mathrm{mL}$. Las recuperaciones se encontraron en el intervalo de 94 a $112 \%$. 
Tabla 1. Recuperaciones de formaldehído en diferentes muestras adicionadas a una concentración de $0,188 \mu \mathrm{g} / \mathrm{mL}$

\begin{tabular}{|c|c|c|c|}
\hline Muestra & $\begin{array}{c}\text { Adicionada } \\
\mu \mathrm{g} / \mathrm{mL}\end{array}$ & $\begin{array}{c}\text { Encontrada } \\
\mu \mathrm{g} / \mathrm{mL}\end{array}$ & $\begin{array}{c}\text { Recuperación } \\
\%\end{array}$ \\
\hline $\mathrm{M} 2$ & 0 & 0,0703 & - \\
\hline & 0,188 & 0,2619 & 102 \\
\hline M7 & 0 & 0,0502 & - \\
\hline & 0,188 & 0,2617 & 112 \\
\hline M17 & 0 & 1,7962 & - \\
\hline & 0,188 & 1,9916 & 104 \\
\hline M22 & 0 & 0,0999 & - \\
\hline & 0,188 & 0,2785 & 95 \\
\hline M37 & 0 & 1,99129 & - \\
\hline & 0,188 & 2,16814 & 94 \\
\hline M42 & 0 & 1,7082 & - \\
\hline & 0,188 & 1,8883 & 96 \\
\hline M47 & 0 & 1,8791 & - \\
\hline & 0,188 & 2,0815 & 108 \\
\hline
\end{tabular}

Fuente: elaboración propia

\section{Discusión}

Los resultados obtenidos en este estudio aplicando el tratamiento propuesto [16] con determinación mediante CLAR revelan que de las 50 muestras de esmaltes de uñas analizadas, el contenido de formaldehído libre fue: $<0,1 \mathrm{mg} / \mathrm{g}$ en treinta muestras; de 0,1 a $0,5 \mathrm{mg} / \mathrm{g}$ en cinco muestras; de 0,5 a $1,0 \mathrm{mg} / \mathrm{g}$ para 11 muestras, y de 1,0 a $1,5 \mathrm{mg} / \mathrm{g}$ en cuatro de las muestras analizadas, con una precisión menor al 5\%, determinada como el RPD. Por otro lado, los valores de recuperación obtenidos en las muestras adicionadas se encontraron en el intervalo de 94 al $112 \%$. Recuperaciones similares fueron obtenidas por Rivero y Topiwala [6], en el análisis de formaldehído libre en muestras de esmaltes de uñas, gel de baño, crema corporal y surfactante con valores de 89 a $101 \%$. Por su parte, Engelhardt y Klinkner [26] obtuvieron 0,295, 0,306, 0,359 mg/g en el análisis de una resina, un esmalte de uñas color rojo y otro transparente, respectivamente.

Por otro lado, al comparar las recuperaciones obtenidas en este trabajo con estudios realizados por otros autores en diferentes matrices, como, por ejemplo, con el realizado por Herrington [24] en muestras de aire aplicando sorbentes sólidos recubiertos con DNFH y medido en un sistema de generación atmosférica dinámico, se obtuvieron recuperaciones de 87 al $101 \%$; mientras que el realizado por Xu et al. [4] en muestras de comida (cerveza de barril, frutas y refresco de cola), obtuvo recuperaciones en el intervalo de 88 a 91,2\%; en el de Nageswari y Krishna [22] de 84 a $97 \%$ en el análisis de fármacos; Storey et al. [19] de $63 \%$ en muestras de bacalao; Ligan et al. [5] de 93,5 a 99,5\% en textiles; en todos estos estudios aplicando derivatización con DNFH y detección por CLAR-UV.

En este contexto, es importante remarcar que la concentración de formaldehído es una función del método de determinación [26], ya que hay técnicas más sensibles con respectos a otras, las cuales proveen de límites de detección bajos y con una alta sensibilidad. Otro factor importante a considerar es el tipo de muestra a analizar (alimentos, cuero, textil, cosméticos, agua, etc.), los cuales pueden causar interferencias de tipo efecto de matriz en el análisis cuantitativo.

\section{Conclusiones}

En este trabajo de investigación se presenta información de relevancia toxicológica, obtenida en la evaluación del formaldehido libre en muestras de esmaltes de uñas comerciales en diferentes marcas. Los resultados señalan que todas las muestras contienen formaldehído por debajo de $2 \mathrm{mg} / \mathrm{g}(0,2 \%)$ -el límite normativo en productos cosméticos-, por lo que se puede observar que el formaldehído se aplica en los esmaltes solo como conservador.

Por otro lado, también se obtuvo información analítica y de factibilidad de aplicación del método Iso 17226-1:2003 con ligeras modificaciones, como un método versátil y robusto que, si bien está validado en muestras de cuero (Iso 2003), también puede ser empleado satisfactoriamente en muestras de textiles [7], o en este caso muestras de esmaltes de uñas con recuperaciones mayores al $90 \%$.

\section{Agradecimientos}

Agradecemos el apoyo brindado por el Consejo de Ciencia y Tecnología del Estado de Guanajuato (CONCYTEG), por la ayuda otorgada para el desarrollo de este proyecto. 


\section{Referencias}

[1] N. G. Yasri, H. Seddik \& M. A. Mosalb, "Spectrophotometric determination of formaldehyde based on the telomerization reaction of tryptamine", Arabian Journal of Chemistry, vol. 8, n. ${ }^{\circ}$ 4, pp. 487-494. 2011. doi: https://doi.org/10.1016/j.arabjc.2011.02.005

[2] Organización Mundial de la Salud, "Formaldehído: Guía para la salud y la seguridad", Programa Internacional de Seguridad de las Sustancias Químicas, n. ${ }^{\circ}$ 57, pp. 2-17, 1995. Disponible en: http://www.bvsde.paho. org/ bvsacd/eco/030511.pdf

[3] Food and Drug Administration. MLM Law: FDA Cosmetics Handbook. Disponible en: http://www.mlmlaw.com/library/guides/fda/Coshdbok.htm

[4] X. Xu, R. Su, X. Zhao, Z. Liu, D. Li, X. Li, et al., "Determination of formaldehyde in beverages using microwave-assisted derivatization and ionic liquid-based dispersive liquid-liquid microextraction followed by high-performance liquid chromatography", Talanta, vol. 85. pp. 2632-2638, 2011. Disponible en: http://www.sciencedirect.com/science/article/pii/ S0039914011007569

[5] L. Chen, H. Jin, L. Wang, L. Sun, H. Xu, L. Ding et al., "Dynamic ultrasound-assisted extraction coupled on-line with solid support derivatization and high performance liquid chromatography for the determination of formaldehyde in textiles", Journal of Chromatography a, vol. 11, n. ${ }^{\circ}$ 92, pp. 89-94, 2008. doi: 10.1016/j.chroma.2008.03.037

[6] R. T. Rivero \& V. Topiwala, "Quantitative determination of formaldehyde in cosmetics using a combined solid-phase microextractión-isotope dilutión mass spectrometry method", Journal of Chromatography A., vol. 1029, pp. 217-222, 2004. doi: https://doi.org/10.1016/j.chroma.2003.12.054

[7] E. T. Martínez-Espinoza, G. Morales-López, J. J. N. Segoviano-Garfías \& M. Maldonado-Santoyo, "Determination of formaldehyde content in footwear samples using a HPLC-Uv technique", International Journal of Scientific and Research Publications, vol. 5, n. ${ }^{\circ} 11$, pp. 225-229, 2015. Disponible en: http://www. ijsrp.org/research-paper-1115.php?rp=P474780

[8] Ch. Lv, J. Sun \& H. Cheng, "Determination of formaldehyde residue in cosmetics by short-column high performance liquid chromatography with mass spectrometric confirmatión", Analytical Methods, vol. 7, pp. 1630-1634, 2015. doi: https://doi.org/10.1039/ C4AY02546F

[9] M. Gandhimathi, M. Jagadeeswaran \& T. K. Ravi, "Evaluation of select cosmetic preparations for the presence of formaldehyde and methyl paraben by novel analytical methods", Indo American Journal of
Pharmaceutical Research, vol. 3, n. ${ }^{\circ}$ 8, pp. 6477-6483, 2013. Disponible en: http://www.scopemed.org/fulltex tpdf.php?mno=154151

[10] Y. Vander-Heyden, A. Nguyen Minh-Nguyet, M. R. Detaevernier, D. L. Massart \& J. Plaizier-Vercammen, "Simultaneous determination of ketoconazole and formaldehyde in shampoo: liquid chromatography method development and validation", Journal of Chromatography A, vol. 958, pp. 191-201, 2002. doi: https://doi.org/10.1016/S0021-9673(02)00384-9

[11] H. Wang, J. Ding, X. Du, L. Chen, Q. Zeng, Y. Xu, et al., "Determination of formaldehide in fruit juice based on magnetic strong cation-exchange resin modified with 2,4-dinitrophenylhydrazine", Food Chemistry, vol. 131, pp. 380-385, 2012. doi: https:// doi.org/10.1016/j.foodchem.2011.08.056

[12] R. J. Elías, V. F. Laurie, S. E. Ebeler, J. W. Wong \& A. L. Waterhouse, "Analysis of select carbonyl oxidation products in wine by liquid chromatography with diode array detection", Analytica Chimica Acta, vol. 626, pp. 104-110, 2008. doi: https://doi. org/10.1016/j.aca.2008.07.048

[13] S. M. Ochs, M. Fasciotti, R. P. Barreto, N. G. de Figueiredo, F. C. Albuquerque, M.C.G. Pontes-Massa et al., "Optimization and comparison of HPLC and RRLC conditions for the analysis of carbonyl-DNFH derivatives", Talanta, vol. 81, pp. 521-529, 2010. doi: 10.1016/j.talanta.2009.12.036

[14] S. Uchiyama, Y. Inaba \& N. Kunugita, "Derivatization of carbonyl compounds with 2,4-dinitrophenylhydrazine and their subsequent determination by high-performance liquid chromatography", Journal of Chromatography B, vol. 879, pp. 1282-1289, 2011. Disponible en: http://www.sciencedirect.com/science/ article/pii/S157002321000615X

[15] D. Bourdin \& V. Desauziers, "Development of SPME on fiber derivatization for the sampling of formaldehyde and other carbonyl compounds in indoor air", Anal Bioanal Chem, vol. 406, pp. 317-328, 2014. Disponible en: http://link.springer.com/article/10.1007\%2Fs00216-013-7460-6

[16] Iso, "Method Iso/Ts 17226:2003 (e): Leather chemical test determinatión of formaldehide content", $1^{\text {a }}$ edición, pp. 1-9, 2003. Disponible en: http://www. iso.org/iso/iso_catalogue/catalogue_ics/catalogue detail_ics.htm?csnumber $=31292$

[17] US-EPA, "Method 8315a: Determination of carbonyl compounds by HIGH performance liquid chromatography (HPLC)", Revisión 1. pp. 1-34, 1996.

[18] W. Pai-Wen, Ch. Chieu-Chen \& Ch. Shin-Shou, "Determination of formaldehyde in cosmetics by HPLC method and acetylacetone method, Journall of food and drug analysis, vol. 11, n. ${ }^{\circ}$ 1, pp. 8-15, 2003. 
Disponible en: http://www.fda.gov.tw/tc/includes/ GetFile.ashx?mID=148\&id=7474\&chk=7bc32085c406-4b65-9964-93fba5e966b8.

[19] J.M.Storey, W.C.Andersen, A.Heise, S. B. Turnipseed, J. Lohne, T. Thomas et al., "A rapid liquid chrmatography determinatión of free formaldehyde in cod". Journal Food additives and contaminants: Part A, vol. 32, no 5, pp. 657-664, 2015. doi: http://dx.doi.org/10 $.1080 / 19440049.2015 .1020530$

[20] A. Soman, Y. Qiu \& Q. Chan-Li, “HPLC-Uv method development and validation for the determination of low level formaldehyde in a drug substance", Journal of Chromatographic Science, vol. 46, pp. 461-465, 2008. Disponible en: http://chromsci.oxfordjournals.org/content/46/6/461.full.pdf

[21] Iris National Acreditation Board, "ps15: Guide to method validation for quantitative analysis in chemical testing laboratories (Iso 17025)", n. ${ }^{\circ} 4$, pp. 1-27, 2016. Disponible en: http://www.inab.ie/ Documents-Forms/Policy/Guide-to-Method-Validation-for-Quantitative-Analysis-in-Chemical-Testing-Laboratories-17025-PDF-36-Pages-349KB-.pdf

[22] A. Nageswari \& K. V. S. R. Krishna Reddy, "Low-level quantification of formaldehyde in drug substance by HPLC-uv, Chromatographia, vol. 75, pp. 275-280, 2012. Disponible en: http://link.springer.com/article/10.1007/s10337-012-2186-8
[23] CENAM \& EMA, Guía técnica sobre trazabilidad e incertidumbre en las mediciones analíticas que emplean las técnicas de cromatografía de gases (CG) y cromatografía de líquidos de alta resolución (CLAR), México, 2008.

[23] J. S. Herrington \& M. D. Hays, "Concerns regarding 24-h sampling for formaldehyde, acetaldehyde, and acrolein using 2,4-dinitrophenylhydrazine (DNFH) coated solid sorbentes", Atmospheric Environment, vol. 55, pp. 179-184, 2012. doi: https://doi. org/10.1016/j.atmosenv.2012.02.088

[24] Us-EPA, Method 8000C:Determinative chromatographic separations. Revisión 3, pp. 1-66, 2003. Disponible en: http://www.caslab.com/EPA-Methods/ PDF/EPA-Method-8000C.pdf

[24] H. Engelhardt \& R. Klinkner, "Determination of free formaldehyde in presence of donators in cosmetics by HPLC and post-column derivation", Chromatographia, vol. 20, n. ${ }^{\circ}$ 9, pp. 559-565, 1985. 